\title{
Role of Intra- and Peritumoral Budding in the Interdisciplinary Management of Rectal Cancer Patients
}

\author{
Inti Zlobec, ${ }^{1}$ Markus Borner, ${ }^{2}$ Alessandro Lugli, ${ }^{1,3}$ and Daniel Inderbitzin ${ }^{4}$ \\ ${ }^{1}$ Institute of Pathology, University of Bern, Murtenstrasse 31, 3010 Bern, Switzerland \\ ${ }^{2}$ Department of Oncology, Hospital Centre Biel, 2502 Bienne, Switzerland \\ ${ }^{3}$ Clinical Pathology Division, Institute of Pathology, University of Bern, Murtenstrasse 31, 3010 Bern, Switzerland \\ ${ }^{4}$ Department of Visceral and Transplantation Surgery, Inselspital-Bern University Hospital, 3010 Bern, Switzerland \\ Correspondence should be addressed to Alessandro Lugli, alessandro.lugli@pathology.unibe.ch
}

Received 11 April 2012; Accepted 23 June 2012

Academic Editor: Ioannis Kanellos

Copyright ( $) 2012$ Inti Zlobec et al. This is an open access article distributed under the Creative Commons Attribution License, which permits unrestricted use, distribution, and reproduction in any medium, provided the original work is properly cited.

The presence of tumor budding ( TuB) at the invasive front of rectal cancers is a valuable indicator of tumor aggressiveness. Tumor buds, typically identified as single cells or small tumor cell clusters detached from the main tumor body, are characterized by loss of cell adhesion, increased migratory, and invasion potential and have been referred to as malignant stem cells. The adverse clinical outcome of patients with a high-grade TuB phenotype has consistently been demonstrated. TuB is a category IIB prognostic factor; it has yet to be investigated in the prospective setting. The value of TuB in oncological and pathological practice goes beyond its use as a simple histomorphological marker of tumor aggressiveness. In this paper, we outline three situations in which the assessment of $\mathrm{TuB}$ may have direct implications on treatment within the multidisciplinary management of patients with rectal cancer: (a) patients with TNM stage II (i.e., T3/T4, N0) disease potentially benefitting from adjuvant therapy, (b) patients with early submucosally invasive ( 1 1, sm 1-sm3) carcinomas at a high risk of nodal positivity and (c) the role of intratumoral budding assessed in preoperative biopsies as a marker for lymph node and distant metastasis thus potentially aiding the identification of patients suitable for neoadjuvant therapy.

\section{Introduction}

Tumor budding (TuB) refers to the presence of detached single tumor cells or clusters of up to 5 cells scattered within the stroma at the invasive tumor front of many different solid cancers [1]. TuB as a histomorphological feature is best described in gastrointestinal tumors and was first comprehensively investigated by Jass in the mid 1980s in patients with rectal cancer [2]. TuB can be evaluated at high magnification using regular $\mathrm{H} \& \mathrm{E}$ staining but its visualization is markedly facilitated with the use of pan-cytokeratin stains (Figure 1).

It is hypothesized that tumor buds, or at least a subpopulation of these cells, have undergone a process similar to epithelial mesenchymal transition (EMT) and have acquired the ability to act as malignant stem cells [3]. Immunohistochemical staining of tumor buds in colorectal cancers shows a clear overexpression of markers involved in extracellular matrix degradation, angiogenesis, migration, and invasion and decreased Ki67 staining indicative of a low proliferation rate [4]. An overexpression of nuclear beta-catenin and simultaneous loss of cell adhesion markers, in particular, Ecadherin is classically observed in tumor-budding cells [5].

With such an aggressive phenotypic constellation, it is not surprising that TuB has consistently been linked to lymph node positivity, the presence of lymphatic and venous invasion, as well as with the presence of distant metastatic disease [6-12]. The frequency of high-grade TuB in colon and rectal cancer varies; it has generally been reported to occur in 25$60 \%$ of all cases but is correlated with disease progression $[7,9,13-15]$. For example, high-grade TuB is reported in $15-17 \%$ of patients with early pT 1 tumors $[8,15,16], 26 \%$ of pT2 cases [17], 36-51\% of pT3 tumors [12, 17, 18], and up to $73 \%$ of pT4 cancers [18]. In addition, it occurs significantly more frequently in patients with node-positive tumors (51$75 \%$ ) in comparison to patients with TNM (6th ed.) stage II 


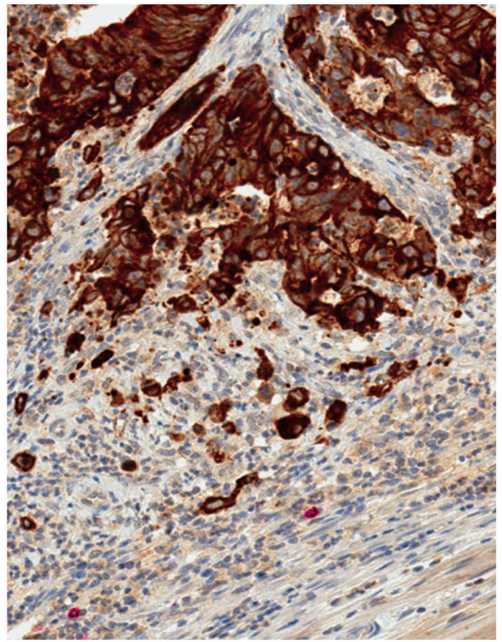

FIGURE 1: Immunohistochemical analysis highlighting the presence of peritumoral buds at the invasion front of rectal cancer (pancytokeratin stain: CK22, 40x magnification).

(T3/T4, N0) (15-29\%) cancers [8, 15, 17]. Currently, TuB is listed as a category IIB prognostic factor and many studies have confirmed that the presence of TuB is associated with poorer overall and disease-specific and disease-free survival, in most cases independently of the TNM stage [19].

The value of TuB in oncological and pathological practice goes beyond its use as a simple histomorphological marker of tumor aggressiveness. In this paper, we outline three situations in which the assessment of TuB may have direct implications on treatment within the multidisciplinary management of patients with rectal cancer. These include (a) the identification of patients with TNM stage II disease potentially benefitting from adjuvant therapy, (b) the identification of patients with early submucosally invasive (T1) carcinomas at a high risk of nodal positivity, and (c) the use of tumor budding as a marker of prognosis and predictor of local and distant relapse assessable in preoperative biopsies.

1.1. Stage II Rectal Cancer Patients. Stage II colorectal cancer patients represent a clinically heterogeneous group. Data from the SEER (1975-2005) Public Use File show 5-year survival trends for patients with colon and rectal cancer [35]. In particular for the latter, 5 -year overall survival rates decrease dramatically from $64.5 \%$ for IIA (T3N0), to $51.6 \%$ for IIB (T4aN0) and $32.3 \%$ for IIC (T4bN0). Generally, patients with stage II colorectal cancer are not typically considered for additional adjuvant therapy without the presence of additional high-risk features such as perforation or venous and lymphatic invasion [36]. It is suggested, however, that a subgroup of patients with stage II disease who would otherwise have unfavorable clinical outcome and high-risk for metastasis may in fact benefit from adjuvant therapy but the identification of such patients using histomorphological or molecular markers is unclear [37].

Although the prognostic effect of high-grade TuB has been well described, studies focusing on the subgroup of stage II cancers are few. Kevans and colleagues evaluated 258 patients with stage II disease and the correlation of TuB with survival and with expression of EMT-related protein markers [38]. They showed that TuB was the only independent marker of poor outcome and had a major effect on the relative risk (RR) of death; patients with high-grade TuB were nearly 8 times more likely to die of disease compared to patients with low-grade TuB. Wang et al. performed a study using 128 patients and evaluated 5-year cancer-specific survival [12]. They show a significant reduction in survival from $91 \%$ to $63 \%$ in patients with low- versus high-grade TuB and a RR of death of 4.76. Nakamura and colleagues studied 5and 10-year survival rates for 200 stage II patients as well as the association of $\mathrm{TuB}$ on the presence of distant metastasis [9]. Again a substantial reduction in 5-year $(93.9 \%$ and $73.9 \%)$ and 10 -year $(90.6 \%$ and $67.8 \%)$ survival time was observed in patients with low-grade versus high-grade $\mathrm{TuB}$ tumors. Tanaka and colleagues confirm this finding, reporting disease-specific survival rates of $98 \%$ versus $74 \%$ in patients with and without TuB, respectively, [10]. Moreover, $\mathrm{TuB}$ in stage II patients has been shown to be independent of other prognostic features $[13,39]$. An increased frequency of liver and peritoneal metastasis was noted in the high-grade TuB group [9]. Earlier studies show that the sensitivity and specificity of high-grade TuB for distant metastatic disease in patients with stage II tumors are 0.76 and 0.739 , respectively, [14]. Frequencies of local recurrence are significantly higher in patients with high- versus low-grade TuB (48\% versus $4.5 \%$, resp.) [10]. Finally, the presence of $\mathrm{TuB}$ has been significantly associated with isolated tumor cells in lymph nodes of patients with stage II disease in both univariate and multivariate analysis [40].

Taken together, these results strongly suggest that $\mathrm{TuB}$ in patients with stage II colorectal cancers has the potential to contribute independent prognostic information. It is linked to more aggressive tumor behavior and is associated with local and distant metastasis. These findings indicate that $\mathrm{TuB}$ should be considered as an important histomorphological parameter and may be worthy of investigation and inclusion in prospective clinical trials of patients with stage II disease.

1.2. Tumor Budding in Early Rectal Cancers. An important issue in the management of patients with submucosally invasive (T1) colorectal carcinomas is the identification of patients after endoscopic resection that may be at "high risk" for lymph node positivity and thus likely to benefit from surgical resection. The rate of lymph node positivity in this setting is low, approximately $10-15 \%[23,24,27,29]$. Nonetheless histomorphological features capable of predicting lymph node involvement are highly sought after.

$\mathrm{TuB}$ has been shown in several studies to have predictive power for lymph node involvement in either univariate or multivariate analyses (Table 1). In addition to other features such as histological type, lymphatic and venous invasion, $\mathrm{TuB}$ is significantly more frequent in cases with lymph node positivity $[22,24-26,28,29]$. One study evaluated the impact of $\mathrm{TuB}$ in $\mathrm{T} 1$ cancers and the potential for the development of distant metastasis. In one subgroup of $\mathrm{T} 1$ patients eventually developing metastatic disease and a control group of 
TABLE 1: Summary of studies evaluating tumor budding in submucosally invasive (T1) colorectal carcinomas.

\begin{tabular}{|c|c|c|c|}
\hline Ref. & Number of patients & Endpoint & Summary of relevant findings \\
\hline$[20]$ & 499 & $\mathrm{LN}+$ & $\begin{array}{l}8.2 \% \text { of T1 were LN+. Several features were independent predictors of LN+: tumor } \\
\text { differentiation/mucinous histology, depth of submucosal invasion, venous invasion, and TuB. }\end{array}$ \\
\hline$[6]$ & 111 & $\mathrm{LN}+$ & $\begin{array}{l}\text { TuB was associated with } \mathrm{LN}+\text { in univariate but not multivariate analysis when analysed with } \\
\text { protein markers. }\end{array}$ \\
\hline$[21]$ & 32 & $\mathrm{DM}$ & $\begin{array}{l}\text { In comparison to a control group, } \mathrm{TuB} \text { was more frequent in patients who eventually had a } \\
\text { distant metastasis in univariate but not multivariate analysis. }\end{array}$ \\
\hline$[22]$ & 111 & $\mathrm{LN}+$ & $\begin{array}{l}\text { Several features were evaluated including lymphatic and venous invasion, submucosal depth, } \\
\text { histologic type, and TuB. In multivariate analysis, only histologic type and TuB predicted LN+. }\end{array}$ \\
\hline [23] & 65 & $\mathrm{LN}+$ & T1-T2 rectal cancers. $6.9 \%$ of $\mathrm{T} 1$ were $\mathrm{LN}+. \mathrm{TuB}$ predicted lateral LN+. \\
\hline$[24]$ & 322 & $\mathrm{LN}+$ & $\begin{array}{l}14.3 \% \text { of } \mathrm{T} 1 \text { were } \mathrm{LN}+\text {. Several features predicted } \mathrm{LN}+\text { : invasion depth, lymphatic and venous } \\
\text { invasion, tumor differentiation, growth pattern, and TuB. Only lymphatic invasion, } \\
\text { differentiation, and TuB were independent predictors in multivariate analysis. }\end{array}$ \\
\hline$[25]$ & 124 & $\mathrm{LN}+$ and $\mathrm{DM}$ & $\begin{array}{l}\text { Elastica von Gieson, D2-40, and CAM5 were used to enhance visualization of venous invasion, } \\
\text { lymphatic invasion, and TuB, respectively. TuB was an independent predictor of LN+ and } \\
\text { DM+ in multivariate analysis. }\end{array}$ \\
\hline$[26]$ & 87 & $\mathrm{LN}+$ and LR & $\begin{array}{l}\text { Prospective study evaluating two groups of patients after endoscopic resection: a surgical } \\
\text { group and a follow-up group without surgery. TuB was the only independent predictor of LN+ } \\
\text { in multivariate analysis. }\end{array}$ \\
\hline$[27]$ & 164 & $\mathrm{LN}+$ & $\begin{array}{l}9.8 \% \text { of } \mathrm{T} 1 \text { were } \mathrm{LN}+\mathrm{TuB} \text { was significantly associated with } \mathrm{LN}+\text { in univariate and } \\
\text { multivariate analysis. }\end{array}$ \\
\hline$[28]$ & 71 & $\mathrm{LN}+$ & $\begin{array}{l}\text { Tumor size, depth of invasion, histologic type, TuB, and lymphatic invasion were predictors in } \\
\text { univariate analysis but only TuB and lymphatic invasion were significant in multivariate } \\
\text { analysis. }\end{array}$ \\
\hline [29] & 86 & $\mathrm{LN}+$ & $\begin{array}{l}13 \% \text { of } \mathrm{T} 1 \text { were } \mathrm{LN}+. \text { Vascular invasion, tumor budding, and degree of submucosal invasion } \\
\text { could be combined to strongly predict LN+. }\end{array}$ \\
\hline$[30]$ & 214 & $\mathrm{LN}+$ & $\begin{array}{l}\text { Several histopathological and protein markers were evaluated. TuB was a significant predictor } \\
\text { in univariate and multivariate analysis. }\end{array}$ \\
\hline$[31]$ & 76 & $\mathrm{LN}+$ & TuB can be used in a predictive equation for $\mathrm{LN}+$. \\
\hline$[32]$ & 56 & $\mathrm{LN}+$ & $\begin{array}{l}\text { TuB evaluated using CAM5 was significantly more frequent in } \mathrm{LN}+(16 / 42) \text { than } \mathrm{LN} \text { negative } \\
(0 / 14) \text { cases. }\end{array}$ \\
\hline$[16]$ & 159 & $\mathrm{LN}+, \mathrm{OS}$ & $\begin{array}{l}10.1 \% \text { of } \mathrm{T} 1 \text { were } \mathrm{LN}+\text { and were associated with several features including TuB. TuB did not } \\
\text { influence overall survival. }\end{array}$ \\
\hline$[33]$ & 51 & $\mathrm{LN}+, \mathrm{LR}$ & TuB was associated to lymphatic invasion, $\mathrm{LN}+$, and local relapse. \\
\hline$[34]$ & 79 & $\mathrm{LN}+$ & $13.9 \%$ were $\mathrm{LN}+. \mathrm{TuB}$ was one of five risk factors for $\mathrm{LN}+$. \\
\hline
\end{tabular}

TuB: tumor budding; LN: lymph node; DM: distant metastasis; LR: local recurrence; OS: overall survival.

T1 patients with favorable long-term outcome, TuB was significantly more frequent in the metastatic cohort [21]. A different study on 145 patients with $\mathrm{T} 1$ cancers used immunohistochemistry and special stains to identify venous invasion, lymphatic invasion, and the presence of TuB by Elastica van Gieson, D2-40 and CAM5 staining, respectively, [25]. In multivariate analysis of lymph node positivity, only venous invasion and $\mathrm{TuB}$ were independently predictive of involvement. TuB could predict the presence of distant metastases but only in univariate analysis.

The examples listed in Table 1 underline the potential importance of the additional assessment of TuB in the pathological diagnosis of early pT1 cancers. TuB assessed in these submucosally invasive carcinomas during daily routine may have a promising role as a histomorphological marker for the prediction of lymph node positivity in this setting.

1.3. Is There a Role for Tumor Budding in the Preoperative Setting? Traditionally, the preoperative rectal biopsy can supply three different types of information. The first is the histopathological diagnosis and confirmation of carcinoma, the second is the histological subtype, and the third is the degree of differentiation (tumor grade). However, recently, studies have not only noted the presence of tumor buds within the preoperative biopsy specimen but have also linked this feature to unfavorable prognostic parameters. We have described the presence of tumor budding within the biopsy specimen as "intratumoral" budding (ITB) in order to distinguish it from the classical "peritumoral" budding (PTB) that is located at the invasive front and thus not normally evaluable in biopsy specimens [41] (Figure 2).

The first assessment of tumor budding in rectal cancer biopsies dates to 1989 [42]. Morodomi and colleagues observed that nearly half of all rectal cancer biopsies contained ITB and its presence was a strong indicator of lymph node positivity. Specifically, lymph node involvement was observed in $78.8 \%$ of ITB-positive cases and in only $28.1 \%$ of ITB-negative rectal cancers. Despite these promising results, 


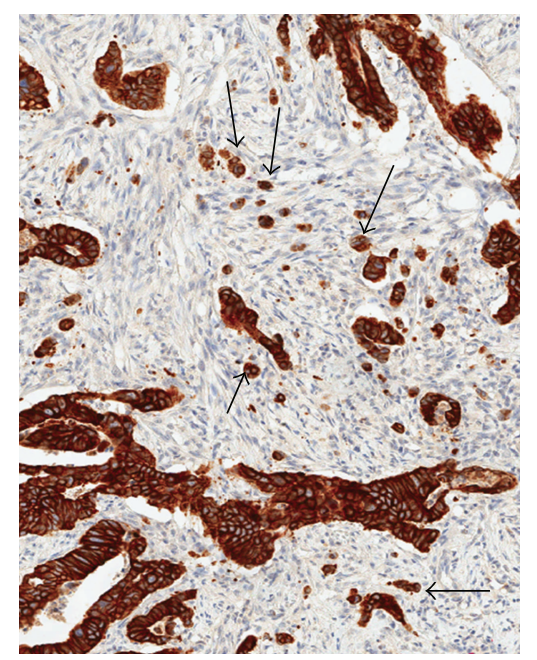

Figure 2: Presence of intratumoral buds (arrows) in the main tumor body of a rectal cancer (pan-cytokeratin stain: CK22, 40x magnification).

the issue of budding within biopsy specimens was only addressed once again in 2011. Using two cohorts of colorectal cancer patients from all stages totaling more than 500 cases, we could confirm the value of ITB as a predictor of lymph node positivity with similar sensitivity $(72.7 \%)$ and specificity $(72.1 \%)$ [41]. The presence of ITB not only correlated with vascular invasion but also showed an independent and unfavorable prognostic effect in multivariable analysis. A recent study by Giger et al. evaluated ITB in preoperative biopsies and the predictive values for both lymph node and distant metastasis in a series of 72 colorectal cancers of all TNM stages [43]. Seventeen percent of all cases were found to have high-grade ITB. Of the ITB positive cases, $83.3 \%$ had lymph node metastasis and $82 \%$ had distant metastasis. This is in contrast to only $51 \%$ and $35 \%$ of ITB-negative cases, respectively.

A strong linear correlation between the presence of ITB in biopsies and corresponding PTB in resection specimens has been made $[41,43]$. This is relevant since the identification of "invasion front" can in some postoperative specimens be challenging. A recent meta-analysis of 42 different histomorphological and immunohistochemical markers in colon and rectal cancers aimed to identify predictors of lymph node metastasis. Focusing on the subset of rectal cancers, Glasgow and colleagues found only two predictive factors, one of which was tumor budding at the invasion front. Again, the sensitivity and specificity of tumor budding for nodepositivity across 7 studies with more than 1500 patients were $70 \%$ and $69.4 \%$ [44].

Taken together, the current body of evidence indicates that regardless of its localization, that is, within the main tumor body or invasion front, tumor budding may be a reliable histomorphological predictor of lymph node metastasis and a factor of poor prognosis which can be applied to both postoperative specimen and, most importantly, preoperative biopsy.

\section{Conclusion}

At least two avenues of investigation should still be clarified before implementing $\mathrm{TuB}$ as a criterion for patient management. First, no prospective studies have been conducted to definitely validate the potential of $\mathrm{TuB}$ in the clinical decision-making process. Secondly, TuB remains severely underreported in daily diagnostic routine due largely to the absence of a standardized or internationally accepted method for its assessment. Nonetheless, efforts are currently on-going to compare and validate the prognostic effects of TuB using various methods of assessment and in particular their interand intraobserver agreement. The evidence supporting an important role of TuB in the clinical and multidisciplinary management of patients with rectal cancer, for example in the setting of stage II and submucosally invasive tumors continues to grow. Although less than a handful of studies have evaluated the presence of intratumoral budding from the preoperative rectal biopsy, the ability to predict, with high accuracy, the presence of lymph node metastases in the pretreatment setting would be of considerable clinical value.

\section{References}

[1] H. Ueno, A. B. Price, K. H. Wilkinson, J. R. Jass, H. Mochizuki, and I. C. Talbot, "A new prognostic staging system for rectal cancer," Annals of Surgery, vol. 240, no. 5, pp. 832-839, 2004.

[2] J. R. Jass, S. B. Love, and J. M. A. Northover, "A new prognostic classification of rectal cancer," The Lancet, vol. 1, no. 8545, pp. 1303-1306, 1987.

[3] T. Brabletz, A. Jung, S. Spaderna, F. Hlubek, and T. Kirchner, "Opinion: migrating cancer stem cells-an integrated concept of malignant tumour progression," Nature Reviews Cancer, vol. 5, no. 9, pp. 744-749, 2005.

[4] I. Zlobec and A. Lugli, "Epithelial mesenchymal transition and tumor budding in aggressive colorectal cancer: tumor budding as oncotarget," Oncotarget, vol. 1, no. 7, pp. 651-661, 2010.

[5] T. Muto, H. Mochizuki, and T. Masaki, Eds., Tumor Budding in Colorectal Cancer, Nova, 2006.

[6] Y. Akishima-Fukasawa, Y. Ishikawa, Y. Akasaka et al., "Histopathological predictors of regional lymph node metastasis at the invasive front in early colorectal cancer," Histopathology, vol. 59, no. 3, pp. 470-481, 2011.

[7] K. Hase, C. Shatney, D. Johnson, M. Trollope, and M. Vierra, "Prognostic value of tumor "budding" in patients with colorectal cancer," Diseases of the Colon and Rectum, vol. 36, no. 7, pp. 627-635, 1993.

[8] A. Lugli, E. Karamitopoulou, I. Panayiotides et al., "CD8+ lymphocytes/ tumour-budding index: an independent prognostic factor representing a pro-/anti-tumour approach to tumour host interaction in colorectal cancer," British Journal of Cancer, vol. 101, no. 8, pp. 1382-1392, 2009.

[9] T. Nakamura, H. Mitomi, S. Kikuchi, Y. Ohtani, and K. Sato, "Evaluation of the usefulness of tumor budding on the prediction of metastasis to the lung and liver after curative excision of colorectal cancer," Hepato-Gastroenterology, vol. 52, no. 65, pp. 1432-1435, 2005.

[10] M. Tanaka, Y. Hashiguchi, H. Ueno, K. Hase, and H. Mochizuki, "Tumor budding at the invasive margin can predict patients at high risk of recurrence after curative surgery for stage II, T3 colon cancer," Diseases of the Colon and Rectum, vol. 46, no. 8, pp. 1054-1059, 2003. 
[11] H. Ueno, H. Mochizuki, Y. Hashiguchi, K. Hatsuse, H. Fujimoto, and K. Hase, "Predictors of extrahepatic recurrence after resection of colorectal liver metastases," British Journal of Surgery, vol. 91, no. 3, pp. 327-333, 2004.

[12] L. M. Wang, D. Kevans, H. Mulcahy et al., "Tumor budding is a strong and reproducible prognostic marker in T3N0 colorectal cancer," American Journal of Surgical Pathology, vol. 33, no. 1, pp. 134-141, 2009.

[13] T. Okuyama, T. Nakamura, and M. Yamaguchi, "Budding is useful to select high-risk patients in stage II well-differentiated or moderately differentiated colon adenocarcinoma," Diseases of the Colon and Rectum, vol. 46, no. 10, pp. 1400-1406, 2003.

[14] F. Prall, H. Nizze, and M. Barten, "Tumour budding as prognostic factor in stage I/II colorectal carcinoma," Histopathology, vol. 47, no. 1, pp. 17-24, 2005.

[15] H. Ueno, J. Murphy, J. R. Jass, H. Mochizuki, and I. C. Talbot, "Tumour "budding" as an index to estimate the potential of aggressiveness in rectal cancer," Histopathology, vol. 40, no. 2, pp. 127-132, 2002.

[16] H. S. Wang, W. Y. Liang, T. C. Lin et al., "Curative resection of T1 colorectal carcinoma: risk of lymph node metastasis and long-term prognosis," Diseases of the Colon and Rectum, vol. 48, no. 6, pp. 1182-1192, 2005.

[17] H. J. Choi, K. J. Park, J. S. Shin, M. S. Roh, H. C. Kwon, and H. S. Lee, "Tumor budding as a prognostic marker in stage-III rectal carcinoma," International Journal of Colorectal Disease, vol. 22, no. 8, pp. 863-868, 2007.

[18] A. L. Canney, D. Kevans, L. M. Wang et al., "Stage II colonic adenocarcinoma: a detailed study of pT4N0 with emphasis on peritoneal involvement and the role of tumour budding," Histopathology. In press.

[19] C. Compton, K. Tanabe, and D. Savarese, "UptoDate: pathology and prognostic determinants of colorectal cancer," 2012.

[20] K. Nakadoi, S. Tanaka, H. Kanao et al., "Management of T1 colorectal carcinoma with special reference to criteria for curative endoscopic resection," Journal of Gastroenterology and Hepatology, vol. 27, no. 6, pp. 1057-1062, 2011.

[21] L. Reggiani Bonetti, C. Di Gregorio, C. de Gaetani et al., "Lymph node micrometastasis and survival of patients with stage I (Dukes' A) colorectal carcinoma," Scandinavian Journal of Gastroenterology, vol. 46, no. 7-8, pp. 881-886, 2011.

[22] K. Komori, T. Hirai, Y. Kanemitsu et al., "Is "depth of submucosal invasion $\geq 1000 \mu \mathrm{m}$ " an important predictive factor for lymph node metastases in early invasive colorectal cancer (PT1)?" Hepato-Gastroenterology, vol. 57, no. 102-103, pp. 1123-1127, 2010.

[23] Y. Homma, T. Hamano, Y. Otsuki, S. Shimizu, H. Kobayashi, and Y. Kobayashi, "Severe tumor budding is a risk factor for lateral lymph node metastasis in early rectal cancers," Journal of Surgical Oncology, vol. 102, no. 3, pp. 230-234, 2010.

[24] Y. Tateishi, Y. Nakanishi, H. Taniguchi, T. Shimoda, and S. Umemura, "Pathological prognostic factors predicting lymph node metastasis in submucosal invasive (T1) colorectal carcinoma," Modern Pathology, vol. 23, no. 8, pp. 1068-1072, 2010.

[25] A. Suzuki, K. Togashi, M. Nokubi et al., "Evaluation of venous invasion by elastica van gieson stain and tumor budding predicts local and distant metastases in patients with $\mathrm{t} 1$ stage colorectal cancer," American Journal of Surgical Pathology, vol. 33, no. 11, pp. 1601-1607, 2009.

[26] D. H. Choi, D. K. Sohn, H. J. Chang, S. B. Lim, H. S. Choi, and S. Y. Jeong, "Indications for subsequent surgery after endoscopic resection of submucosally invasive colorectal carcinomas: a prospective cohort study," Diseases of the Colon and Rectum, vol. 52, no. 3, pp. 438-445, 2009.
[27] H. Yamauchi, K. Togashi, Y. J. Kawamura et al., "Pathological predictors for lymph node metastasis in T1 colorectal cancer," Surgery Today, vol. 38, no. 10, pp. 905-910, 2008.

[28] Y. Ishikawa, Y. Akishima-Fukasawa, K. Ito et al., "Histopathologic determinants of regional lymph node metastasis in early colorectal cancer," Cancer, vol. 112, no. 4, pp. 924-933, 2008.

[29] K. Yasuda, M. Inomata, A. Shiromizu, N. Shiraishi, H. Higashi, and S. Kitano, "Risk factors for occult lymph node metastasis of colorectal cancer invading the submucosa and indications for endoscopic mucosal resection," Diseases of the Colon and Rectum, vol. 50, no. 9, pp. 1370-1376, 2007.

[30] I. Kaneko, S. Tanaka, S. Oka et al., "Immunohistochemical molecular markers as predictors of curability of endoscopically resected submucosal colorectal cancer," World Journal of Gastroenterology, vol. 13, no. 28, pp. 3829-3835, 2007.

[31] T. Masaki, H. Matsuoka, M. Sugiyama, N. Abe, A. Sakamoto, and Y. Atomi, "Actual number of tumor budding as a new tool for the individualization of treatment of $\mathrm{T} 1$ colorectal carcinomas," Journal of Gastroenterology and Hepatology, vol. 21, no. 7, pp. 1115-1121, 2006.

[32] S. Kazama, T. Watanabe, Y. Ajioka, T. Kanazawa, and H. Nagawa, "Tumour budding at the deepest invasive margin correlates with lymph node metastasis in submucosal colorectal cancer detected by anticytokeratin antibody CAM5.2," British Journal of Cancer, vol. 94, no. 2, pp. 293-298, 2006.

[33] T. Masaki, M. Sugiyama, H. Matsuoka et al., "Clinical utility of grading criteria for submucosal invasion in the prognosis of T1 colorectal carcinomas," Journal of Gastroenterology, vol. 38, no. 1, pp. 37-44, 2003.

[34] K. Hase, C. H. Shatney, H. Mochizuki et al., "Long-term results of curative resection of "minimally invasive" colorectal cancer," Diseases of the Colon and Rectum, vol. 38, no. 1, pp. 19-26, 1995.

[35] S. Edge, D. Byrd, C. Compton, A. Fritz, F. Greene, and A. Trotti, Eds., AJCC Cancer Staging Manual, Springer, New York, NY, USA, 7th edition, 2010.

[36] A. de Gramont, B. Chibaudel, A. K. Larsen, C. Tournigand, and T. Andre, "The evolution of adjuvant therapy in the treatment of early-stage colon cancer," Clinical Colorectal Cancer, vol. 10, no. 4, pp. 218-226, 2011.

[37] C. C. Compton, "Optimal pathologic staging: defining stage II disease," Clinical Cancer Research, vol. 13, no. 22, part 2, pp. 6862s-670s, 2007.

[38] D. Kevans, L. M. Wang, K. Sheahan et al., "Epithelial-mesenchymal transition (EMT) protein expression in a cohort of stage II colorectal cancer patients with characterized tumor budding and mismatch repair protein status," International Journal of Surgical Pathology, vol. 19, no. 6, pp. 751-760, 2011.

[39] V. Koelzer, M. Horcic, L. Terracciano, I. Zlobec, and A. Lugli, "Tumour budding score based on 10 high-power fields (HPFs) is a reliable basis for a standardized scoring system in colorectal cancer," Virchows Archiv, vol. 459, supplement 1, article S156, 2011.

[40] S. Y. Park, G. Choe, H. S. Lee, S. Y. Jung, J. G. Park, and W. H. Kim, "Tumor budding as an indicator of isolated tumor cells in lymph nodes from patients with node-negative colorectal cancer," Diseases of the Colon and Rectum, vol. 48, no. 2, pp. 292-302, 2005.

[41] A. Lugli, T. Vlajnic, O. Giger et al., "Intratumoral budding as a potential parameter of tumor progression in mismatch repairproficient and mismatch repair-deficient colorectal cancer patients," Human Pathology, vol. 42, no. 12, pp. 1833-1840, 2011. 
[42] T. Morodomi, H. Isomoto, K. Shirouzu, K. Kakegawa, K. Irie, and M. Morimatsu, "An index for estimating the probability of lymph node metastasis in rectal cancers. Lymph node metastasis and the histopathology of actively invasive regions of cancer," Cancer, vol. 63, no. 3, pp. 539-543, 1989.

[43] O. Giger, S. Comtesse, A. Lugli, I. Zlobec, and M. Kurrer, "Intra-tumoral budding (ITB) in pre-operative biopsy specimens predicts lymph node and distant metastasis in patients with colorectal cancer," Modern Pathology, vol. 25, no. 7, pp. 1048-1053, 2012.

[44] S. C. Glasgow, J. I. Bleier, L. J. Burgart, C. O. Finne, and A. C. Lowry, "Meta-analysis of histopathological features of primary colorectal cancers that predict lymph node metastases," Journal of Gastrointestinal Surgery, vol. 16, no. 5, pp. 1019-1028, 2012. 


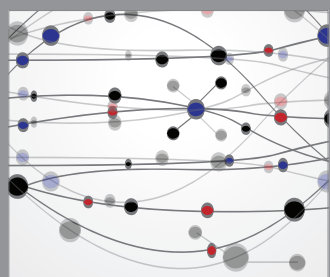

The Scientific World Journal
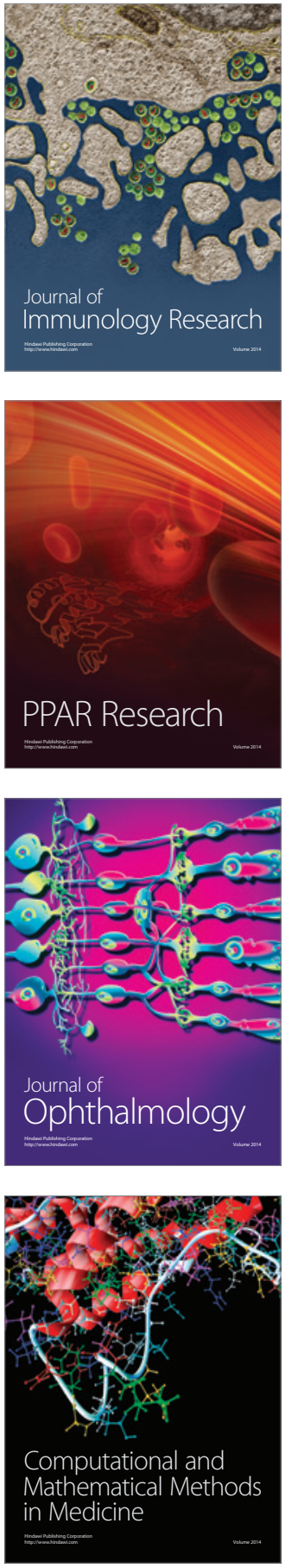

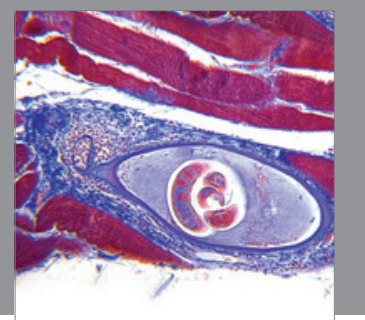

Gastroenterology

Research and Practice
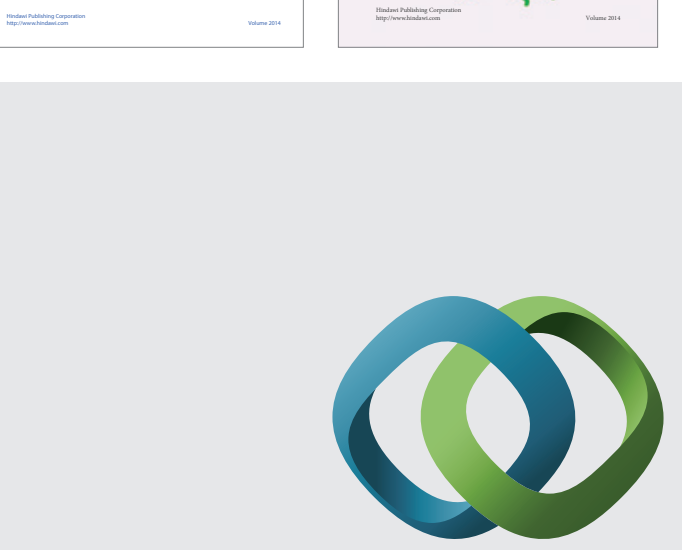

\section{Hindawi}

Submit your manuscripts at

http://www.hindawi.com
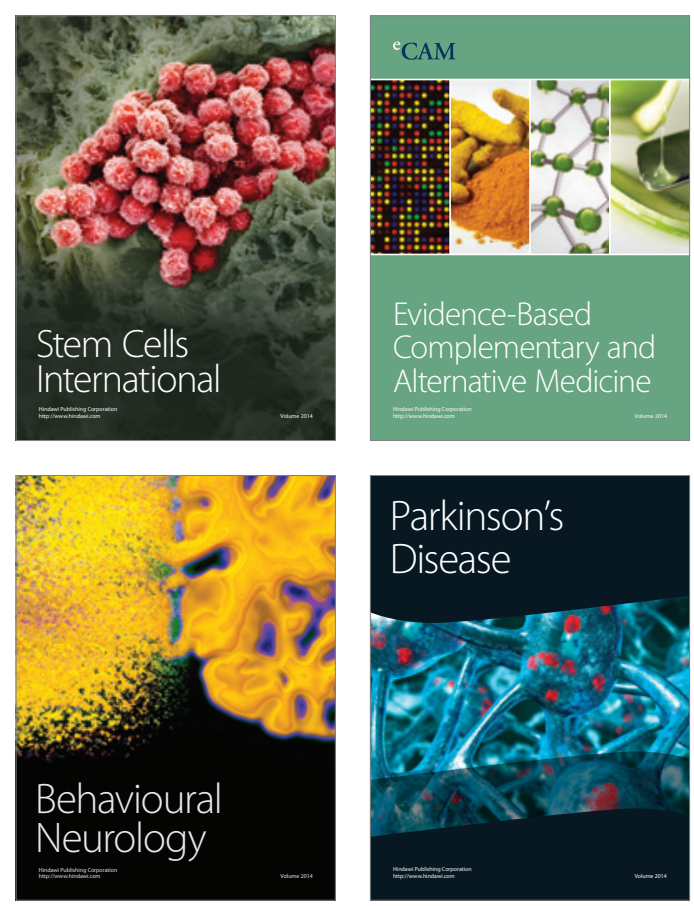

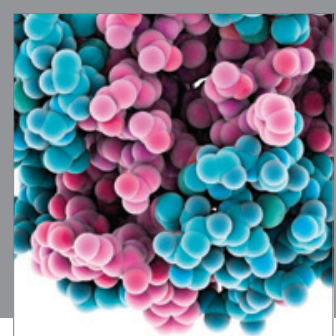

Journal of
Diabetes Research

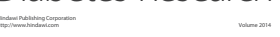

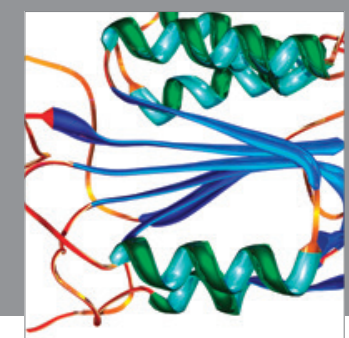

Disease Markers
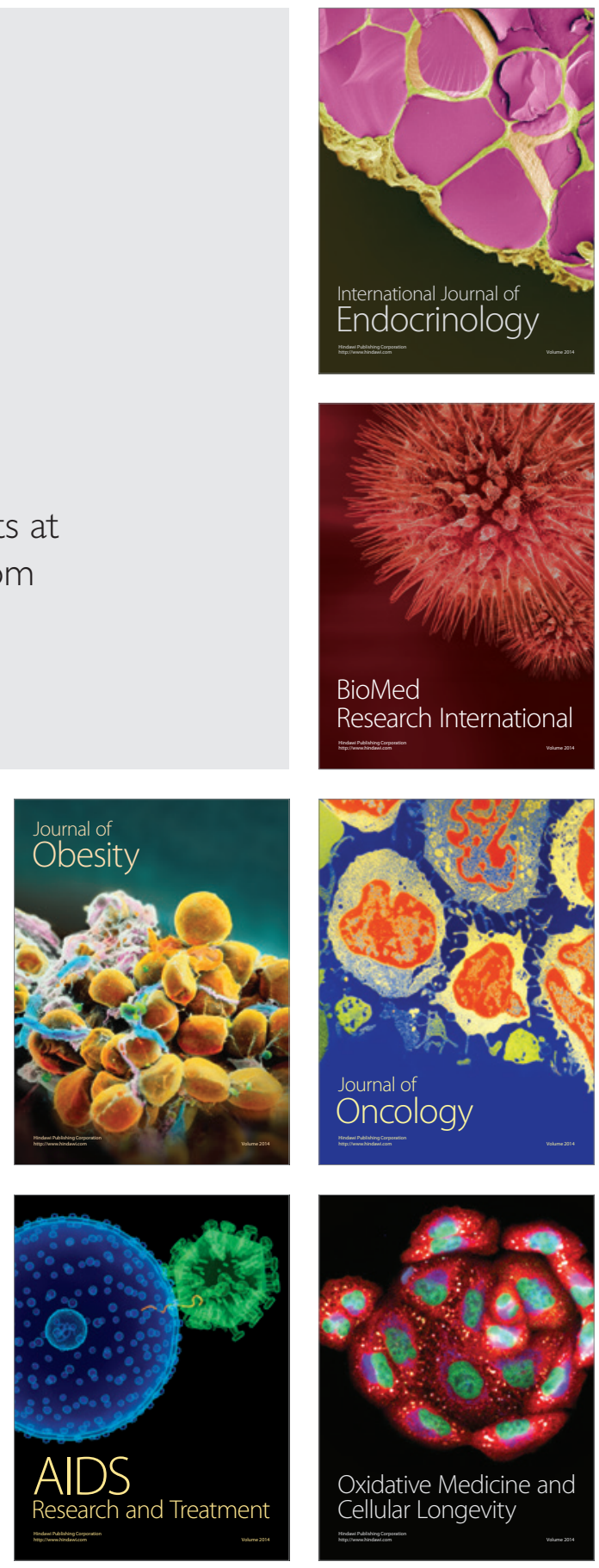